Der Physik-Preis 2010 wurde Frau Corinna Kollath, Palaiseau/Frankreich, in Anerkennung ihrer Arbeiten zur „Dynamik quantenmechanischer Vielteilchensysteme weit weg vom Gleichgewicht" verliehen.

\title{
Dynamik in ultrakalten Atomgasen
}

\section{Corinna Kollath}

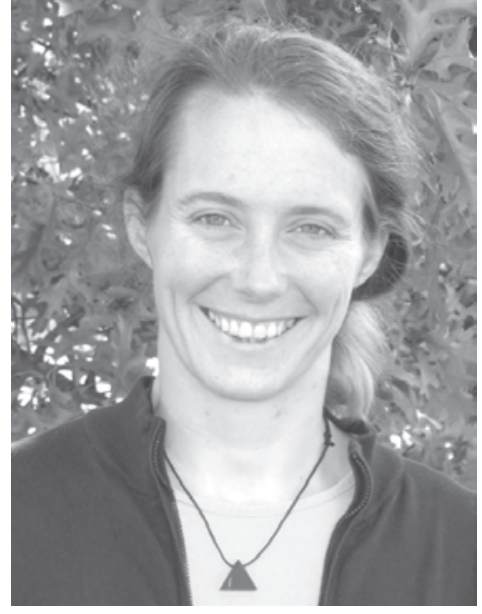

Corinna Kollath, Chargé de Recherche (CNRS) im Centre de Physique Théorique der Ecole Polytechnique (Palaiseau) und Chaire Junior, Triangle de la Physique in Frankreich, Trägerin des Physik-Preises 2010

Wellen hervorruft?

\section{Einleitung}

Ultrakalte Atome bilden einen der kältesten Plätze im Universum. Atome werden heutezutage in mehreren Laboren auf dieser Erde durch Licht abgebremst und eingefangen. Da die sehr kalten Atome durch die Lichtfelder im Vakuum gehalten werden, haben sie kaum Kontakt zu ihrer Umgebung. Was passiert, wenn man diese normalerweise gut isolierten Atome stört? Wie verhält sich ein einzelnes Atom, wenn man es in einen "See“ von anderen ultrakalten Atomen einbringt? Behält dieses Teilchen seinen Teilchencharakter und stößt mit den anderen Atomen wie Bälle (Abbildung 1)? Oder verhält es sich wie ein Tropfen, der ins Wasser fällt und nur kollektive

Wir werden diese Fragestellung im Folgenden in eindimensionalen stark wechselwirkenden Quantengasen, d. h. in sehr kalten Gasen, diskutieren und die Aufspaltung des Teilchens in kollektive Anregungen, die sogenannte Spinladungstrennung, finden.

Das Verständnis und die Kontrolle dieser Quantendynamik ist nicht nur von fundamentalem theoretischen Interesse, sondern wird eine entscheidende Rolle für den zukünftigen technologischen Fortschritt spielen. Ein Beispiel ist die Miniaturisierung technischer Geräte wie zum Beispiel des Computers. Schon in heutigen Computern beruht die Speicherung 


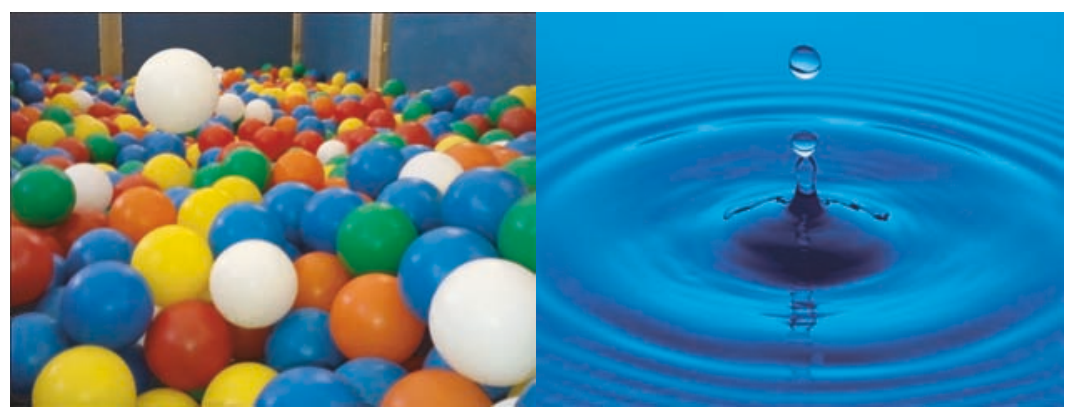

Abbildung 1: links: Ein Ball fällt in ein Meer von Bällen (B. Middelberg), rechts: Ein Tropfen fällt ins Wasser (PhotoDisc, Inc).

eines Bits, der kleinsten Einheit der Daten, auf wenigen hundert Elektronen. Diese Entwicklung wird in nächster Zukunft an ihre Grenzen stoßen, wenn nicht Quantenbausteine eingesetzt werden können, in denen schon einzelne Elektronen kontrolliert werden.

\section{Das Bose-Einstein-Kondensat}

In den letzten Jahrzehnten haben es Forscher geschafft, Atome mit der Hilfe von intensivem Laserlicht und starken magnetischen Feldern zu zähmen. Ultrakalte Atomgase werden mit optischen oder mit magnetischen Feldern im Ultrahochvakuum gefangen und sind daher nahezu perfekt von der Umgebung isoliert. Der Fortschritt, Laserlicht zu verwenden, um Atome zu fangen und zu kühlen, hatte derart weite Auswirkungen, dass im Jahre 1997 ein Nobelpreis für diese Entdeckung verliehen wurde. In den folgenden Jahren konnten Atome in Kombination mit anderen Techniken zu vorher unvorstellbar tiefen Temperaturen, einem millionstel Grad über dem absoluten Nullpunkt, gekühlt werden. Bei solch tiefen Temperaturen kommen Quanteneffekte ans Licht.

Die Temperatur eines solchen atomaren Gases kann durch die Geschwindigkeit der Atome charakterisiert werden: Bei Raumtemperatur bewegen sich die Atome mit Geschwindigkeiten von mehreren hundert Metern pro Sekunde. Diese Geschwindigkeiten sind ähnlich denen von Flugzeugen. Nach dem Kühlprozess hingegen sind die Atome fast bis zum Stillstand hin abgebremst. Ihre Durchschnittsgeschwindigkeit - wenige Millimeter pro Sekunde - ist langsamer als die einer Schnecke. Bei derart tiefen Temperaturen können bosonische Atome nicht mehr als einzelne Teilchen betrachtet 


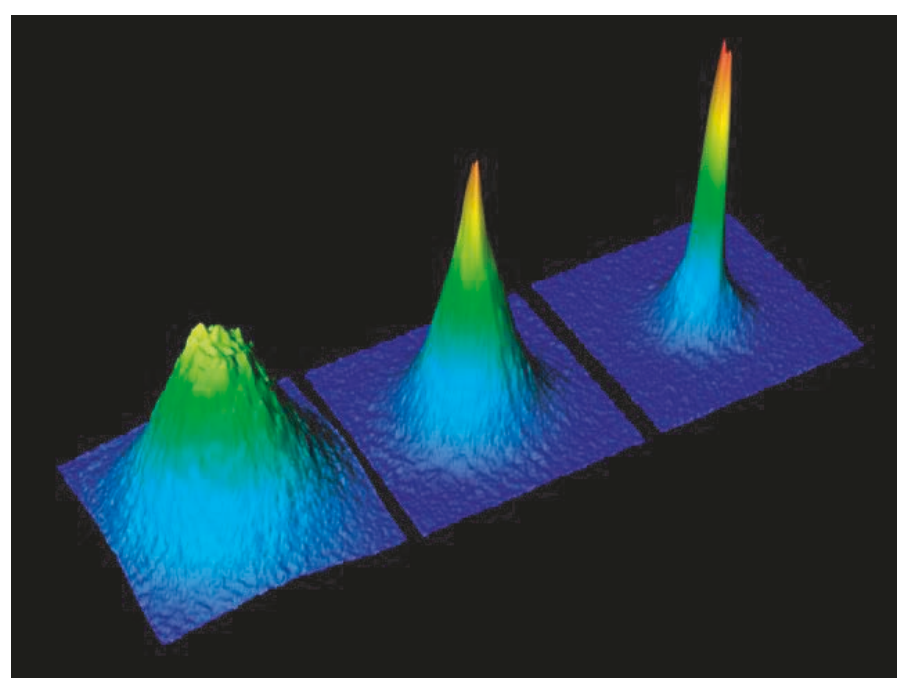

Abbildung 2: Geschwindigkeitsverteilung kalter bosonischer Gase beim Kühlen bis zur Bose-Einstein-Kondensation. Die Temperatur sinkt von links nach rechts. (T. Esslinger, ETH Zürich).

werden, sondern bilden einen kollektiven Zustand, das sogenannte BoseEinstein-Kondensat. Diesen kollektiven Zustand, in dem eine makroskopische Anzahl von Atomen im selben Quantenzustand sind, kann durch eine grosse Welle verbildlicht werden. Damit konnte endlich der Effekt der Kondensatbildung, der von Bose und Einstein vor mehr als 80 Jahren vorhergesagt worden ist, im Labor realisiert werden.

In Abbildung 2 zeigen wir den experimentellen Nachweis eines BoseEinstein-Kondensats. In den verschiedenen Teilfiguren wird die gemessene Geschwindigkeitsverteilung der Atome bei verschiedenen Temperaturen gezeigt. Die erst noch sehr breite Geschwindigkeitsverteilung entwickelt sich mehr und mehr zu einer sehr starken Spitze, die das Bose-EinsteinKondensat signalisiert. In einem solchen Bose-Einstein-Kondensat, für dessen Herstellung 2001 der Nobelpreis verliehen wurde, hat man eine sehr gute Kontrolle über die Atome, so dass viele Grundsatzfragen untersucht werden können.

\section{Dynamik in eindimensionalen Systemen}

Zusätzlich können mit optischen, laserinduzierten Potentialen atomare Gase auf der Nanometerskala strukturiert werden. Dazu gehören künstliche 


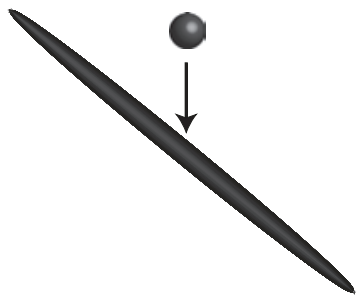

Abbildung 3: Skizze des Hinzufügens eines einzelnen Atoms mit Teilchencharakter zu einer Flüssigkeit von Atomen.

periodische Potentiale, sogenannte optische Gitter, die durch entgegengesetzt laufende Laserstrahlen gebildet werden, und niedrigdimensionale Strukturen. Für die niedrigdimensionalen Strukturen wird der extrem starke Einschluss des optischen Potentials ausgenutzt, um die Atombewegung auf eine oder zwei Dimensionen zu beschränken.

Niedrigdimensionale Systeme weisen viele unerwartete Effekte auf. Eindimensionale klassische Systeme haben die Besonderheit, dass die Teilchen sich nicht aneinander vorbeibewegen können. Diese Besonderheit kann man sehr einfach verstehen, wenn man sich Bälle in einem Rohr anschaut. In einem engen Rohr können die Bälle sich nur hintereinander her bewegen. Sobald ein Ball stoppt, können die anderen nicht vorbei. Ist es dagegen ein breites Rohr, können die Bälle aneinander vorbei. Daher kann die Dynamik der Bälle sich in den beiden Situationen sehr unterscheiden. Genauso treten in Quantensystemen wichtige Effekte durch die Einschränkung der Bewegungsfreiheit auf.

In einem eindimensionalen kalten Gas verhalten sich Atome wie Wellen statt wie einzelne Teilchen. Man nennt diesen Zustand auch die LuttingerFlüssigkeit, in dem es keine teilchenartigen Anregungen mit der üblichen Dispersion $\mathrm{E}(\mathrm{k}) \propto \mathrm{k}^{2}$ mehr gibt, sondern nur kollektive Anregungen wie zum Beispiel Dichtewellen mit linearer Dispersion $\mathrm{E}(\mathrm{k}) \propto \mathrm{k}$.

Ein sehr unintuitiver und faszinierender Effekt taucht auf, wenn ein Atom aus einem separaten Reservoir zu einer Luttinger-Flüssigkeit hinzugebracht wird (Einteilchenanregung) (siehe Abbildung 3).

Generell trägt ein Atom zwei Eigenschaften: seinen Spin, der auf oder ab zeigen kann, und seine Ladung für geladene Teilchen bzw. seine Dichte für neutrale Atome ${ }^{1}$. Wenn es zu den anderen Atomen gebracht wird, verliert es seinen Teilchencharakter. Es verschmilzt mit den anderen Atomen und

\footnotetext{
$\overline{1}$ Wir verwenden im folgenden aus historischen Gründen das Wort Ladung, auch wenn wir
} neutrale Atome betrachten. 


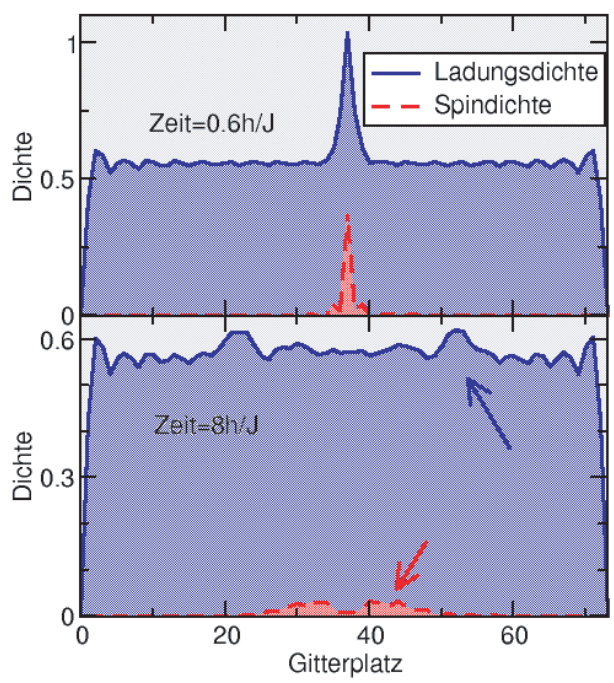

Abbildung 4: Spinladungstrennung im sogenannten Hubbard-Modell, einem sehr wichtigen Modell in der Quantenphysik. Momentaufnahmen der Zeitentwicklung der Spinund der Ladungsdichte. Zum Zeitpunkt $\mathrm{t}=0$ wurde eine Einteilchenanregung in der Mitte erzeugt, die sich in kollektive Spin- und Ladungsanregung auftrennt (siehe Pfeile). Die Existenz von zwei entgegengesetzt propagierenden Anregungen sowohl in der Spinals auch in der Ladungsdichte ist eine Folge der Symmetrie des betrachteten Systems.

formt zwei kollektive Wellenanregungen, eine im Spin- und eine im Ladungssektor. Diese beiden Anregungen bewegen sich mit unterschiedlichen Geschwindigkeiten, so dass nach kurzer Zeit die Spin- und die Ladungswellen voneinander getrennt sind. Dieses Phänomen wird Spinladungstrennung genannt.

Der beschriebene Effekt steht im Gegensatz zu dem Verhalten in einem typischen System mit drei Dimensionen. Darin behält ein hinzugefügtes fermionisches Teilchen für eine gewisse Zeit seinen Teilchencharakter. Es bewegt sich als sogenanntes Quasiteilchen. Die Eigenschaften des Quasiteilchens können durch die Anwesenheit der anderen Atome geringfügig verändert werden, jedoch trägt es immer wie das ursprüngliche Teilchen sowohl Ladung als auch Spin.

Die Trennung von Spin und Ladung für sehr lange Zeiten kann durch die sogenannte Luttingerflüssigkeitstheorie, einen analytischen Zugang für den Bereich niedriger Energien ${ }^{2}$, beschrieben werden.

2 K. Schönhammer, Interacting Electrons in Low Dimensions, Kluwer Academic Publishers (2003), T. Giamarchi, Quantum Physics in One Dimension, Oxford University Press (2004). 
Erst vor wenigen Jahren ist es uns gelungen, die Trennung von Spin und Ladung nach dem Hinzufügen eines fermionischen Teilchens für realistische Systemgrössen (ca. hundert Plätze) zu simulieren ${ }^{3}$. Die numerischen Ergebnisse in Abbildung 4 zeigen eine deutliche Trennung von Spin und Ladung. Sie geben insbesondere Aufschluss über die Zeitskalen, auf denen diese Auftrennung erfolgt. Weiter zeigen sie, dass die Spinladungstrennung als charakteristische Eigenschaft eindimensionaler Systeme über den Bereich kleiner Anregungen hinaus erhalten bleibt.

\section{Zusammenfassung und Ausblick}

Die Quantendynamik von starkkorrelierten Vielteilchensystemen führt zu überraschenden Effekten. Wir haben einen davon, die Spinladungstrennung in eindimensionalen Systemen erörtert. Ultrakalte Quantengase ermöglichen heutezutage eine experimentelle Untersuchung dieser Dynamik in einer sehr kontrollierten Weise. Viele spannende Fragestellungen warten darauf, theoretisch und experimentell untersucht zu werden.

\section{Danksagungen}

Ich möchte mich an dieser Stelle bei der Akademie der Wissenschaften zu Göttingen für die Auszeichnung und die Gelegenheit bedanken, mein Arbeitsgebiet vorzustellen. Weiter danke ich Prof. Ulrich Schollwöck, Prof. Jan von Delft, Prof. Wilhelm Zwerger, Prof. Thierry Giamarchi und Prof. Antoine Georges für eine fruchtbare Zusammenarbeit und ihre anwährende Unterstützung.

Herausheben möchte ich auch die finanziellen Beiträge des „Triangle de la Physique" und des ANR (FAMOUS).

3 C. Kollath, U. Schollwöck und W. Zwerger, Phys. Rev. Lett. 95, 176401 (2005). C. Kollath, Physik Journal 8, 40 (2009). 